\title{
COMPARATIVE STUDY ON EFFICACY OF AMOXICILLIN WITH CLAVULANIC ACID VERSUS AZITHROMYCIN IN TONSILLITIS
}

Ruchi Shrestha ${ }^{1}$, Sirisa Karki ${ }^{2}$, Tejendra Manandhar ${ }^{3}$, Sushma Deo ${ }^{4}$

\begin{abstract}
INTRODUCTION

Tonsillitis is the most commonly encountered health related problems in the general population. Good hydration, use of analgesic along with antibiotics is important for treatment. Penicillin was recommended as the first line treatment for bacterial tonsillitis but now has been replaced mostly by newer antibiotics. This study was attempted to compare the efficacy of amoxicillin with clavulanic acid (AMC) and azithromycin in tonsillitis.
\end{abstract}

\section{MATERIAL AND METHODS}

Prospective, randomized study was carried out in 70 patients of tonsillitis visiting Department of Otorhinolaryngology, Nepal Medical College and Teaching Hospital (NMCTH), Attarkhel, Kathmandu, Nepal. After clinical assessment and recording of baseline sign and symptoms score, patients were randomized into 2 groups. First group received amoxicillin with clavulanic acid and second group received azithromycin. Efficacy of both drugs was compared by reduction in the sign and symptoms score in tonsillitis on 6th day. i.e. after five days of treatment.

\section{RESULTS}

Both drug groups demonstrated statistically significant reduction in sign and symptoms score after 5 days of treatment when compared from baseline. However, the clinical efficacy of amoxicillin with clavulanic acid and azithromycin did not differ significantly $(\mathrm{P}>0.05)$.

\section{CONCLUSION}

This study suggests that both the antibiotics, amoxicillin with clavulanic acid and azithromycin had similar efficacy at the end of 5 days treatment.

KEY WORDS Tonsillitis, Comparative, Amoxicillin with clavulanic acid, Azithromycin

1. Lecturer, Department of Pharmacology, Universal College of Medical Sciences, Bhairahawa, Nepal

2. Lecturer, Department of Pharmacology, Chitwan Medical College, Bharatpur, Chitwan, Nepal

3. Lecturer, Department of Pharmacology, KIST Medical College, Lalitpur, Nepal

4. Lecturer, Department of Pharmacology, Gandaki Medical College, Pokhara, Nepal

\author{
For Correspondence \\ Dr. Ruchi Shrestha \\ Lecturer \\ Department of Pharmacology \\ Universal College of Medical Sciences \\ Bhairahawa, Nepal \\ Email: ruchistha11@gmail.com
}




\section{INTRODUCTION}

Tonsillitis is inflammation of the tonsils most commonly caused by bacterial or viral infection. ${ }^{1}$ It is one of the most common problem encountered by otorhinolaryngologists in the pediatric population and adult population. ${ }^{2,3}$ This condition is responsible for more than 6 million physician office visits annually by children in 5-15 years of age group. ${ }^{4}$

Tonsillitis occurs either due to bacterial or viral infection. The most common causative organism for bacterial tonsillitis is Group A beta hemolytic streptococcus (GABHS). ${ }^{5}$ The prevalence of GABHS induced tonsillitis is $15 \%$ to $30 \%$ of children with sore throat and $5 \%$ to $15 \%$ of adults with sore throat. $^{6-8}$ GABHS causes several suppurative and non suppurative infections and is important cause of morbidity and mortality worldwide every year.

In general, tonsillitis runs its course without complications and heals within three to four days. Rare but serious complications after tonsillitis are glomerulonephritis and rheumatic fever. ${ }^{10}$ A second, more frequent complication is peritonsillar abscess. ${ }^{11}$ In clinically definite or proven bacterial tonsillitis associated with distress, antibiotic therapy is justified. It shortens the course of disease, reduces fever and pain compared to placebo. In addition, antibiotic therapy provides relatively reliable protection against the rheumatic fever and glomerulonephritis, which often leads to arthritis, myocarditis and death. ${ }^{12}$

Treatment of acute tonsillitis include penicillin, penicillin congeners (ampicillin or amoxicillin), clindamycin, certain cephalosporin and macrolides. ${ }^{13}$ Tonsillectomy is the choice of treatment in chronic and recurrent cases. ${ }^{14}$ For tonsillar abscess, drainage of abscess followed by antibiotics and surgery is recommended. ${ }^{10}$ The combination of amoxicillin with clavulanic acid (AMC) has excellent activity against gram positive bacteria, including GABHS. ${ }^{15}$ Azithromycin is effective against most common upper respiratory bacterial pathogens such as GABHS and also has a good safety profile. ${ }^{16}$ It offer better pharmacokinetic/pharmacodynamic properties with less frequent daily dosing and reduced gastrointestinal tract (GIT) side effects. ${ }^{17}$

As per records in Nepal Medical College and Teaching Hospital (NMCTH), tonsillitis has high prevalence rate and AMC and azithromycin are frequently prescribed antibiotics for tonsillitis. This study attempted to compare the efficacy of $\mathrm{AMC}$ and azithromycin in tonsillitis patients attending Department of Otorhinolaryngology in NMCTH.

Aim of the study is to evaluate and compare efficacy of amoxicillin with clavulanic acid and azithromycin

\section{MATERIAL AND METHODS}

This hospital based randomized, prospective study was conducted in patients of tonsillitis attending in Department of Otorhinolaryngology in NMCTH. Ethical clearance was taken from the ethical review committee of NMCTH. Duration of study was October 2013-September 2014 and sample size included 70 patients. Inclusion criteria for the study was patients aged 5 to 44 years clinically diagnosed as bacterial tonsillitis by concerned ENT consultant. Exclusion criteria included patients clinically diagnosed as viral tonsillitis by concerned ENT consultant, patients with history of hypersensitivity reaction or who developed hypersensitivity reaction to AMC, azithromycin during course of treatment, pregnant and lactating mother, patients with severe condition requiring admission.

Data was collected using pretested proforma. Each patient was included in the study after obtaining informed consent. The demographic information (age, sex, address, occupation) and clinical information (duration, sign and symptoms scores of tonsillitis), drug prescribed, dose, severity of the disease was recorded in proforma. All patients fulfilling the inclusion criteria were randomized into two groups: Group A and Group B. Odd number patients were included in Group A and even number patients in Group B. Group A received AMC 625mg TDS for 5 days and for pediatric patients, the dose was $20-40 \mathrm{mg} / \mathrm{kg} /$ day TDS for 5 days. Group B received azithromycin 500mg OD for 5 days and for pediatric patients, the dose was $10 \mathrm{mg} / \mathrm{kg}$ /day OD for 5 days. Along with test drug, patients were also prescribed NSAIDS, mouthwashes according to need. Clinical efficacy of the study drugs, AMC and azithromycin were compared on the basis of sign and symptoms scores where maximum value was 3 . Sign and symptoms score was observed on baseline (day 1 ) and on $6^{\text {th }}$ day. i.e. after 5 days of treatment in both drug groups. Sign and symptoms scoring included three parameters: sore throat, dysphagia and tonsillar hypertrophy.

The used score helped to determine the severity of disease i.e. absent, mild, moderate, severe. Severity of disease was graded as '0'-absent, '1'-mild, '2'-moderate, '3'-severe. After treatment, a decrease in the clinical sign and symptoms to a total severity of score 0 or 1 (from score 3 ) and score 0 (from score 2 and 1 ) was considered clinical cure.

For sore throat

Score 0: absent; Score 1: mild (less severe than cold); Score 2: moderate (like cold); Score 3 :severe (more severe than cold)

For dysphagia

Score 0: absent; Score 1: mild (painful with certain foods); 
Score 2: moderate (painful with all foods); Score 3: severe (unable to swallow own secretions)

For tonsillar hypertrophy

Score 0: absent (tonsils inside tonsillar fossa); Score 1: mild (tonsils occupying upto $25 \%$ of oropharynx); Score 2: moderate (tonsils occupying upto $26-50 \%$ of oropharynx); Score 3: severe (tonsils occupying more than 50\% of oropharynx) Statistical analysis of all the collected data was carried out using Statistical Package for Social Sciences (SPSS) version 20. The data was analyzed for demographic data of patients and clinical efficacy of drugs. Descriptive statistics like Chi-square test was used to analyze socio-demographic data. Statistical test of significance like paired $t$ test and independent sample $t$ test were applied to find $\mathrm{P}$ value. The $\mathrm{P}$ value less than 0.05 was considered statistically significant, less than 0.01 as highly statistically significant and less than 0.001 as very highly statistically significant.

\section{RESULTS}

Out of 82 tonsillitis patients enrolled in the study, seven patients experienced severe diarrhoea, gastric upset while the remaining 5 did not take the medicine for five consecutive days. So, altogether twelve patients were excluded from the study. Therefore, a total of 70 patients were included in the study from which 35 patients each were randomly assigned to AMC and azithromycin groups. Out of 70 patients, 30 $(42.9 \%)$ were male and $40(57.1 \%)$ were female. The mean age of the patients under study was $21.35 \pm 1.02$ years (range 5-44 years). The most commonly involved age group was 514 years $(35.7 \%)$. The results of age and sex distribution of tonsillitis patients are summarized in table 1 .

Table 1. Age and gender wise distribution of tonsillitis patients

\begin{tabular}{|c|c|c|c|c|c|c|}
\hline \multirow[t]{3}{*}{ Age group (years) } & \multicolumn{4}{|c|}{ Gender } & \multirow{2}{*}{\multicolumn{2}{|c|}{ Total }} \\
\hline & \multicolumn{2}{|c|}{ Male } & \multicolumn{2}{|c|}{ Female } & & \\
\hline & $\mathrm{n}$ & $\%$ & $\mathbf{n}$ & $\%$ & $\mathrm{n}$ & $\%$ \\
\hline 5-14 & 11 & 15.7 & 14 & 20 & 25 & 35.7 \\
\hline $15-24$ & 8 & 11.4 & 11 & 15.7 & 19 & 27.1 . \\
\hline $25-34$ & 8 & 11.4 & 11 & 15.7 & 19 & 27.1 \\
\hline $35-44$ & 3 & 4.4 & 4 & 5.7 & 7 & 10.1 \\
\hline Total & 30 & 42.9 & 40 & \begin{tabular}{|l|l|}
57.1 \\
\end{tabular} & 70 & 100 \\
\hline
\end{tabular}

$(\mathrm{n}=$ number of subjects)

Clinical efficacy of the drugs, AMC and azithromycin were compared on the basis of reduction in sign and symptoms score from baseline (day 1 ) to $6^{\text {th }}$ day. i.e. after 5 days of treatment. Maximum sign and symptoms score was 3. On day one (baseline), the mean score for sign and symptoms in
AMC group was $1.94 \pm 0.73$ and after treatment, the mean sign and symptoms score was reduced to $0.49 \pm 0.85$. Similarly, in azithromycin group, the baseline mean score for sign and symptoms was $2.02 \pm 0.66$ and after treatment, the mean score was reduced to $0.66 \pm 0.90$. There was very highly statistically significant reduction $(\mathrm{P}=0.000$ i.e. $\mathrm{P}<0.001)$ in mean sign and symptoms scores after treatment as compared to day one in AMC group whereas there was highly statistically significant reduction $(\mathrm{P}=0.006$ i.e. $\mathrm{P}<0.01)$ in mean sign and symptoms scores after treatment as compared to day one in azithromycin group. The results obtained on mean sign and symptoms scores in tonsillitis before and after treatment in amoxicillin group is shown in table 2 and in azithromycin group is shown in table 3 .

Table 2. Sign and symptoms score before and after treatment in AMC group

\begin{tabular}{|c|c|c|c|}
\hline \multirow{2}{*}{ Drug group } & \multicolumn{2}{|c|}{ Sign and symptoms score } & \multirow{2}{*}{ P value } \\
\cline { 2 - 3 } & Before treatment & After treatment & \\
\hline AMC & $1.94 \pm 0.73$ & $0.49 \pm 0.85$ & $0.000(<0.001)$ \\
\hline
\end{tabular}

Table 3. Sign and symptoms score before and after zithromycin group treatment in a azithromycin group

\begin{tabular}{|l|c|c|c|}
\hline \multirow{2}{*}{ Drug group } & \multicolumn{2}{|c|}{ Sign and symptoms score } & \multirow{2}{*}{ P value } \\
\cline { 2 - 3 } & Before treatment & After treatment & \\
\hline Azithromycin & $2.02 \pm 0.66$ & $0.66 \pm 0.90$ & $0.006(<0.01)$ \\
\hline
\end{tabular}

Although there was very highly statistically significant reduction in sign and symptoms scores by both individual drugs, but at the end of study, the comparison between two drug groups demonstrated that there was no difference in the efficacy of two drugs on these scores. When the mean sign and symptoms score between two drug groups were compared from baseline (on day 1), no statistically significant difference was observed (table 4). P value was 0.41 ( $\mathrm{P}>0.05)$. Similarly, when the mean sign and symptoms score between two study drugs were compared after treatment, no statistically significant difference in sign and symptoms score was observed. (table 5). P value was 0.13 $(>0.05)$.

Table 4. Comparison of sign and symptoms score in both drugs before treatment

\begin{tabular}{|l|c|c|c|}
\hline Visit day & \multicolumn{2}{|c|}{ Drug group } & \multirow{2}{*}{ P value } \\
\cline { 2 - 3 } & AMC & Azithromycin & \\
\hline Day one & $1.94 \pm 0.73$ & $2.02 \pm 0.66$ & $0.41(>0.05)$ \\
\hline
\end{tabular}


Table 5. Comparison of sign and symptoms score in both drugs after treatment

\begin{tabular}{|c|c|c|c|}
\hline \multirow{2}{*}{ Visit day } & \multicolumn{2}{|c|}{ Drug group } & \multirow{2}{*}{ P value } \\
\cline { 2 - 3 } & AMC & Azithromycin & \\
\hline After treatment & $0.49 \pm 0.85$ & $0.66 \pm 0.90$ & $0.13(>0.05)$ \\
\hline
\end{tabular}

After treatment, a decrease in the clinical sign and symptoms to a total severity of score 0 or 1 (from score 3 ) and score 0 (from score 2 and 1) was considered clinical cure. When cure rates between two drugs were compared after 5 days of treatment, it was found that in amoxicillin with clavulanic acid group, cure rate was $88.6 \%$ whereas in azithromycin group, cure rate at the end of the treatment was $71.4 \%$.

\section{DISCUSSION}

Tonsillitis is among the most commonly encountered health related problems in the general population. ${ }^{18}$ Tonsillitis may be caused by a variety of microorganisms. GABHS induced tonsillitis may lead to suppurative and nonsuppurative complications such as rheumatic fever and acute glomerulonephritis. ${ }^{19,20}$ Antimicrobial therapy are given to reduce the severity and duration of symptoms, reduce the incidence of suppurative and non suppurative complications and to reduce the transmission. ${ }^{21}$

In this study, comparative study on clinical efficacy of AMC versus azithromycin in tonsillitis was attempted. A prospective and randomized study was conducted in Department of Otorhinolaryngology, NMCTH from October 2013 to September 2014. Seventy patients of tonsillitis were included. The obtained data was statistically analyzed.

In the present study, the number of male and female patients suffering from tonsillitis were 30 and 40 respectively. The female patients were 1.33 times higher than male patients in the study. The result obtained in the present study is in agreement with the study conducted by Abouzied et $\mathrm{al}^{22}$ where female patients were 1.6 times higher than male patients. Similarly, in the study conducted by Thorp, ${ }^{23}$ significant predominance of female cases of tonsillitis were seen in which female to male ratio was 3.22:1. The study conducted by Kishve et $\mathrm{al}^{24}$ revealed the same conclusion in which number of female patients was 1.11 times higher than male patients in throat disorders. Therefore, the result obtained in the present study is supported by earlier studies.

In this study, the incidence of tonsillitis was higher in the age group 5-14 years (25 patients). The mean age of the patients was $21.35 \pm 1.02$ years (range $5-44$ years). The result obtained was somewhat similar to study conducted by Agrawal et $\mathrm{al}^{25}$ who found the commonest age group of tonsillitis is between
11-20 years. In the study conducted by Gunnarsson, ${ }^{26}$ it was reported that highest prevalence rate of tonsillitis was in the age group 3-15 years. Similarly, in the study conducted by Jha et $\mathrm{al}^{27}$ the most common age group recorded was 15 to 25 . Therefore, the result of this study corroborates the reports by other workers who reported that the incidence of tonsillitis is higher in younger age.

All patients in this study were evaluated for the degree of sore throat, dysphagia and tonsillar hypertrophy. Sign and symptoms score scale where maximum value was 3 , the mean sign and symptoms score at day one for AMC group was $1.94 \pm 0.73$ and on follow up after receiving 5 days treatment, it reduced to $0.49 \pm 0.85$. While the mean sign and symptoms score at day one for azithromycin group was $2.02 \pm 0.66$ and on follow up after receiving 5 days treatment, it was reduced to $0.66 \pm 0.90$. There was very highly statistically significant reduction $(\mathrm{P}<0.001)$ in signs and symptoms score by AMC and highly statistically significant reduction $(\mathrm{P}<0.001)$ in signs and symptoms score by azithromycin. However, after 5 days of treatment, the comparison between two groups revealed that there was no statistically significant difference in sign and symptoms score $(\mathrm{P}>0.05)$. The cure rate after 5 days course of treatment in amoxicillin with clavulanic acid group was $88.6 \%$ and in azithromycin group it was $71.4 \%$. Increased resistance could be reason for less cure rate in azithromycin group due to its widespread dispense from pharmacy, overuse and misuse by patients because of its less frequent dosing.

Different studies have been done to compare efficacy of AMC and azithromycin in foreign countries. In the study done by Bottaro et al, ${ }^{28}$ it was concluded that 3 days azithromycin course was more effective than 7-14 days of therapy with AMC. In the study done by Lauvau et al, ${ }^{29}$ it was suggested that 3 days of azithromycin course was as effective as 5-10 days regimen of AMC. However, in the study done by Casey et $\mathrm{al}^{30}$ it was found that 10 -day courses of amoxicillin and cephalosporin were statistically superior to the 5-day course of azithromycin.

\section{CONCLUSION}

Tonsillitis in which the main causative agent is GABHS, usually require antibiotic therapy to shorten duration of illness and to reduce complications. In this study, two antibiotics, AMC and azithromycin were compared for their efficacy in tonsillitis. Both study drugs were effective in the treatment of tonsillitis. The efficacy of both AMC and azithromycin were not significantly different in 5 days treatment period. In this study, sample size was small. Therefore, multicenter studies with large sample size are required. This study was not based on microbiological cure 
rates. So, further studies are warranted to assess both clinical and microbiological cure rates. This study did not assess the adverse effects of the drugs. So, further studies are warranted to assess both efficacy and adverse effect of these drugs.

\section{REFERENCES}

1. Sih TM, Bricks LF. Optimizing the management of the main acute infections in pediatric ORL: Tonsillitis, Sinusitis, Otitis media. Braz J Otorhinolaryngol 2008;74(5):755-62.

2. Weinberger PM, Terris DJ. Otolaryngology-head \& neck surgery. In: Doherty GM, editor. Current Diagnosis and Treatment: Surgery, 13th edition. New York: McGraw-Hill 2010 : 224-58.3.Loganathan A, Arumainathan UD, Raman R. Comparative study of bacteriology in recurrent tonsillitis among children and adults. Singapore Med J 2006;47(4):271-5.

4. Schappert SM, Burt CW. Ambulatory care visits to physician offices, hospital outpatient departments and emergency departments: United States, 2001-2. Vital Health Stat 2006 ;159:1-66.

5. Shenoy PK. "Acute Tonsillitis"-if left untreated could cause severe fatal complications. J Curr Clin Care 2012;2(4):28-34.

6. Komaroff AL, Pass TM, Aronson MD, Ervin CT, Cretin S, Winickoff RN, et al. The prediction of streptococcal pharyngitis in adults. J Gen Intern Med 1986;1(1):1-7.

7. Kaplan EL, Top FH, Dudding BA, Wannamaker LW. Diagnosis of streptococcal pharyngitis: differentiation of active infection from the carrier state in the symptomatic child. J Infect Dis 1971;123(5):490-501.

8. Schroeder BM. Diagnosis and management of group A streptococcal pharyngitis. Am Fam Physician 2003;67(4):8834.
9. Carapetis JR, Steer AC, Mulholland EK, Weber M. The global burden of group Astreptococcal diseases. Lancet Infect Dis 2005;5(11):685-94

10. Feinstein AR, Levitt $M$. The role of tonsils in predisposing to streptococcal infections and recurrences of rheumatic fever. $\mathrm{N}$ Engl J Med 1970;282(6):285-91.

11. Segal N, El-Saied S, Puterman M. Peritonsillar abscess in children in the southern district of Israel. Int $\mathrm{J}$ Pediatr Otorhinolaryngol 2009;73(8):1148-50

12. Stelter K. Tonsillitis and sore throat in children. GMS Curr Top Otorhinolaryngol Head Neck Surg 2014;13.

13. Brook I, Dohar JE. Management of group A $\beta$-hemolytic streptococcal pharyngotonsillitis in children. J Fam Pract 2006 ;55(12):1-11

14. Discolo CM, Darrow DH, Koltai PJ. Infectious indications for tonsillectomy. Pediatr Clin N Am 2003;50(2):445-58

15. Todd PA, Benfield P. Amoxicillin/clavulanic acid: an update of its antibacterial activity, pharmacokinetic properties and therapeutic use. Drugs 1990;39(2):264-307.

16. Jain $\mathrm{N}$, Lodha $\mathrm{R}$, Kabra SK. Upper respiratory tract infections. Indian J Pediatr 2001;68(12):1135-8.

17. Blondeau JM. Update on the use of the macrolides for community acquired respiratory tract infections. Therapy 2006 ;3(5):619-50 